\title{
Long-stay patients discharged from psychiatric
}

\section{hospitals}

\author{
Social and clinical outcomes after five years in the community. \\ The TAPS Project 46
}

\section{JULIAN LEFF and NOAM TRIEMAN}

\author{
Background There have been no \\ large-scale prospective studies evaluating \\ the transfer of care from psychiatric \\ hospitals to district-based services.
}

Aims We aimed to compare the quality of life of patients in two north London hospitals scheduled for closure with that in the community homes to which they were discharged.

Method The total long-stay population of Friern Hospital and several hundred long-stay patients in Claybury Hospital were assessed with a batch of eight schedules while in hospital. They were followed up after one year in the community and then at five years.

Results Of the 670 discharged patients, 126 died before the five-year follow-up. Data were obtained on 523 (97\%) of the survivors. There was no change in the patients' clinical state or in their problems of social behaviour. However, they gained domestic and community living skills. They also acquired friends and confidants. They were living in much freer conditions and the great majority wanted to remain in their current homes.

\section{Conclusions Community care has} enhanced the quality of life of this group of patients, involved in a well-planned and adequately resourced reprovision programme.

\section{Declaration of interest TAPS} research was funded by the Department of Health, NorthThames Regional Health Authority, and the Sainsbury Family Trusts.
Of the 130 psychiatric hospitals which were operating in England and Wales in 1975, more than 90 have now been closed. The Team for the Assessment of Psychiatric Services (TAPS) was established in 1985 by the North East Thames Regional Health Authority (NETRHA) to evaluate the policy of replacing psychiatric hospitals with district-based services. One of TAPS' major projects has been to follow up long-stay non-demented patients discharged from Friern and Claybury Hospitals, two of the six psychiatric hospitals managed by NETRHA. Friern Hospital closed on schedule in March 1993 after a 10-year reprovision programme, while the closure of Claybury was delayed until January 1997 because of financial constraints. Consequently, it was possible to follow up the total long-stay population of Friern Hospital but only a proportion of the equivalent Claybury patients. Our one-year follow-up (Leff $e t$ al, 1996) showed a generally beneficial outcome for the majority of the patients. However, nearly half the patients had been in hospital for more than 20 years (O'Driscoll \& Leff, 1993) and a longer follow-up was clearly desirable, so TAPS mounted a five-year follow-up of the whole sample.

\section{REVIEW OF THE LITERATURE}

There have been few long-term follow-up studies of patients discharged after long periods of stay in psychiatric hospitals. Most of the previous studies were either short-term (Pickard et al, 1992) or confined to small selected samples (Braun et al, 1981). The largest and longest study was by Harding et al (1987), who conducted a 32-year follow-up of 269 patients discharged from Vermont State Hospital, USA, in the 1950s. The sample was not representative of the hospital population at the time, as patients from back wards were selected to take part in an experimental rehabilitation programme. The findings were that over half the patients were functioning much better than had been originally predicted for them. Comparisons over time were restricted by the fact that assessments were only standardised well into the study.

Other long-term follow-up studies, such as those by Bleuler (1972) and Ciompi (1980), have not included long-stay patients, for the reason that such patients were not considered suitable for discharge in the era in which the samples were chosen. This type of study was aimed at determining the natural course of schizophrenia rather than assessing the impact of community care.

We have previously published a preliminary report on the five-year findings for the 114 patients discharged in the first two years of the reprovision programme (Leff et al, 1994). Here we present the clinical and social outcomes of the five-year followup of the total sample of 670 patients. The administrative outcomes are presented elsewhere (Trieman et al, 1999).

\section{METHOD}

\section{Subjects}

The criteria for entry into the study were a length of stay of one year or more and, for those over the age of 65 , the absence of a diagnosis of dementia. A total of 770 patients in the two hospitals met these criteria when TAPS conducted a census in 1985 . Over the next 10 years, while Friern and Claybury Hospitals continued to admit patients, some stayed more than a year and technically became 'long-stay'. The accumulation of these 'new long-stay' patients swelled the sample, but the death of other patients before they could be discharged decreased it. The final sample comprised 670 patients, consisting of all 470 long-stay patients discharged to the community from Friern Hospital between 1985 and its closure in 1993, plus 200 patients from Claybury Hospital discharged between 1985 and 1990, when its reprovision programme was temporarily halted. Data from the baseline assessments were used to compare the samples from the two hospitals and remarkably few differences emerged (O’Driscoll \& Leff, 1993). The Friern sample does not include a group of 57 patients who were transferred to other hospitals or a residual group of 64 'difficult-to-place' patients who were relocated in specialised 
facilities and are the subject of a separate study (Trieman \& Leff, 1996).

\section{Design}

Patients were assessed at three points in time with a batch of eight schedules described below. All patients were assessed at baseline and those who had not died or been lost from the study were assessed at one-year and five-year follow-ups. Patients who were in hospital at the time of a follow-up were also included, although some of the schedules were omitted as not relevant.

The batch of schedules is described in detail by O'Driscoll \& Leff (1993). The Personal Data and Psychiatric History Schedule was used to record demographic and historical data obtained from the case notes. The patients' mental state was assessed with the Present State Examination (PSE) (Wing et al, 1974). Problems of social behaviour were derived from interviews with staff using the Social Behaviour Schedule (SBS) (Wykes \& Sturt, 1986) and this information was supplemented with data from the Basic Everyday Living Skills Schedule (BELS). Problems with the patients' physical health and treatments given were recorded with the Physical Health Index (PHI) from information in the case notes and from interviews with the care staff. Patients' attitudes to the care received and to the care environment were ascertained with the Patient Attitude Questionnaire (PAQ) (Thornicroft et al, 1993). The degree of restrictiveness of the patients' living situation was measured with the Environmental Index (EI) while the extent and quality of their social networks were assessed with the Social Network Schedule (SNS; Leff et al, 1990).

\section{Reliability of assessments}

The interview schedules are of established reliability. Many different researchers collected the data over the 13 years of this study. Each new member of the team was trained to administer and rate the batch of schedules in a standardised training programme that involved live interviews with patients who were not part of the sample. Trainees had to reach an interrater reliability of over 0.8 on all schedules before being allowed to collect data on the study patients. In order to prevent drift from the established standards of interviewing and rating, interrater reliability exercises using non-study patients were held with all the current interviewers every two weeks throughout the duration of the study.

\section{Follow-up}

In addition to the visits by the researchers to each patient for the one-year and five-year follow-ups, the TAPS administrator made six-monthly telephone calls in rotation to every facility in which study patients resided, to check whether they still lived there and to collect information on hospital admissions and deaths. Supplementary information was obtained from hospital records and case notes, and by contacting agencies such as local social services and the National Health Service (NHS) Central Register.

\section{Data analysis}

Some patients refused or were unable to respond to individual questions or whole schedules at one or more of the three assessment points. Other patients died or were lost before the one-year or five-year follow-up. The aim was to detect changes in each response variable over one and five years, while utilising all available data at each of the three time points and taking into account the lack of independence, or within-patient correlations. The method of analysis utilised the general estimating equations (GEE) approach of Zeger \& Liang (1986) (Diggle et $a l, 1994)$. This allows the marginal distributions at each time point to be modelled while controlling for within-subject correlations, using appropriate covariance structure. The method was implemented using the software package SPIDA (Gebski et al, 1992), which allows for various within-subject correlation structures to be selected. The method chosen was defined as 'exchangeable', in which correlation between observations at different times is assumed to be the same. This structure allows for unbalanced data within cases (necessary for missing data). Results were compared with those obtained using other correlation assumptions and were generally found to differ very little.

Where data were not normally distributed, suitable transformations of the raw data, such as square-root or log, were necessary to induce normality. For analysis of dichotomous response variables involving proportions, a binomial error distribution was assumed. In order to minimise chance findings, 16 variables were selected for analysis on the basis of our experience with the full one-year outcome data and the preliminary five-year outcome data.
In addition to the analysis of changes over time, multiple linear regression analyses were conducted to investigate the relationship between baseline variables and the outcome after five years in the community. These analyses enable one to determine the simultaneous effect of each factor while controlling for the effects of all the other factors. The baseline variables studied were: age, gender, number of previous admissions, original/new long-stay patient, diagnosis, provider of accommodation, and the nature of the home discharged to (staffed/unstaffed, and household size).

\section{RESULTS}

\section{Characteristics of patients}

At baseline assessment in the two hospitals the mean age of the total sample was 54 years (range 19-97). Just over half of the group were men, and only $6 \%$ were currently married. Ethnic minorities formed $12 \%$ of the sample. The median length of continuous stay in hospital was 28 years for Claybury patients and 21 years for those from Friern. However, new long-stay patients constituted one-third of the sample. The most frequent diagnosis $(80 \%)$ was schizophrenia, and the most common social behaviour problem was poor hygiene (59\%). The overall level of functioning was low (O'Driscoll \& Leff, 1993), with the least disabled patients being selected for discharge earlier (Jones, 1993).

\section{Success of follow-up}

Of the 670 long-stay patients discharged into the community, 126 died during the five-year follow-up, 12 patients moved too far out of London to be assessed and nine could not be traced. The remaining 523 patients were successfully followed up throughout the five years, representing $97 \%$ of those who had not died.

\section{Clinical outcome}

\section{Present State Examination}

Four variables from the PSE were analysed: the total score, and scores for delusions and hallucinations (DAH), negative symptoms (PSENEG), and behaviour, observed affect and speech (BSO) (see Table 1). In the tables that follow, the data are presented in the form of estimates from the GEE. Time 1 is the baseline, and Times 2 and 3 the one-year and five-year follow-ups. It can be seen that there is an overlap of the 
Table I Estimated values over time for the Present State Examinatin total score and sub-scores

\begin{tabular}{|c|c|c|c|}
\hline Time point $^{1}$ & $n$ & Score & $95 \% \mathrm{Cl}$ \\
\hline & & PSE total score & \\
\hline I & 631 & II.I & $10.3-11.9$ \\
\hline 2 & 525 & 10.3 & $9.4-11.2$ \\
\hline \multirow[t]{2}{*}{3} & 392 & 10.0 & $9.0-11.0$ \\
\hline & & $\%$ with DAH score $>$ I & \\
\hline I & 631 & 41.2 & $37.4-45.1$ \\
\hline 2 & 525 & 39.0 & $35.0-43.2$ \\
\hline \multirow[t]{2}{*}{3} & 392 & 42.8 & $38.1-47.4$ \\
\hline & & PSENEG & \\
\hline I & 651 & I.II & $1.02-1.20$ \\
\hline 2 & 538 & I.II & $1.00-1.21$ \\
\hline \multirow[t]{2}{*}{3} & 398 & 1.00 & $0.90-1.11$ \\
\hline & & BSO & \\
\hline I & 631 & 2.77 & $2.54-2.99$ \\
\hline 2 & 525 & 2.57 & $2.33-2.83$ \\
\hline 3 & 392 & 2.66 & $2.36-2.97$ \\
\hline
\end{tabular}

I. Time points I, 2 and 3 =baseline, one-year follow-up and five-year follow-up.

DAH, delusions and hallucinations sub-score; PSENEG, negative symptoms sub-score; BSO, behaviour, observed affect and speech sub-score.

Table 2 Estimated values over time for variables from the Social Behaviour Schedule (SBS) and Behaviour and Everyday Living Skills Schedule (BELS)

\begin{tabular}{lccc}
\hline Time point $^{1}$ & $n$ & Score & $95 \% \mathrm{Cl}$ \\
\hline & & SBSTOT & \\
\cline { 3 - 4 } & & 3.53 & $3.27-3.81$ \\
2 & 664 & 3.28 & $3.01-3.57$ \\
3 & 551 & 3.27 & $2.98-3.57$ \\
& 435 & & $1.13-1.30$ \\
1 & & SBSNEG & $1.05-1.23$ \\
3 & 670 & 1.22 & $0.96-1.16$
\end{tabular}

BELS community skills

\begin{tabular}{rrr}
395 & 6.91 & $6.45-7.36$ \\
547 & 8.21 & $7.82-8.61$ \\
435 & 7.87 & $7.45-8.30$ \\
& BELS domestic skills & \\
395 & 10.2 & $9.3-10.8$ \\
544 & 14.6 & $13.6-15.9$ \\
433 & 11.5 & $10.6-12.4$ \\
\hline
\end{tabular}

I. Time points I, 2 and 3 =baseline, one-year follow-up and five-year follow-up. SBSTOT, SBSNEG, total score, and sub-score for behaviour attibuted to negative symptoms. estimated confidence limits for the three time points for the total score of the PSE, indicating no significant change over time. The same is true of the three other PSE variables. It is noteworthy that about $40 \%$ of the patients with a completed PSE suffered from active delusions and/or hallucinations throughout the follow-up period.

\section{Social outcome}

\section{Social Behaviour Schedule}

Two variables from this schedule were analysed: the total score (SBSTOT) and the score for behaviour attributed to negative symptoms (SBSNEG) (see Table 2). There was no change over time in social behaviour problems as a whole or in problems attributed to negative symptoms.

\section{Behaviour and Everyday Living Skills Schedule}

This schedule was introduced into the batch of assessments only after the first year of the study. Consequently, a smaller number of patients was assessed at baseline than at the follow-ups. Two variables from this schedule were analysed: the score for community skills and the score for domestic skills (see Table 2). Community skills had increased significantly by the one-year followup and this improvement was maintained over the whole five years. Domestic skills also showed a significant improvement at one year, but had then dropped back somewhat by five years, although the overlap of confidence levels with the baseline value is minimal.

\section{Social Network Schedule}

Three variables were analysed: the total number of named social contacts in the previous month (TOTNAME), the number of contacts whom patients identified as friends (SUMFREN) and the number of contacts in whom they felt they could confide (SUMCONF) (see Table 3). The size of patients' networks did not change over the five years of follow-up. However there was a significant increase in the number of friends in the first year which was sustained over the five-year period. The number of confidants had increased between baseline and one year, and even more over the next four years, differing significantly from the baseline level after five years in the community. 
Table 3 Estimated values over time for variables from the Social Network Schedule

\begin{tabular}{llcc}
\hline Time point $^{\prime}$ & $n$ & & $95 \% \mathrm{Cl}$ \\
\hline & & TOTNAME & \\
\hline & & 7.78 & $7.13-8.46$ \\
2 & 505 & 8.81 & $8.08-9.56$ \\
3 & 409 & 7.39 & $6.56-8.29$ \\
& 286 & SUMFREN & \\
1 & & 2.33 & $2.02-2.66$ \\
2 & 505 & 3.68 & $3.23-4.17$ \\
3 & 409 & 3.39 & $2.87-3.96$ \\
& 286 & SUMCONF & $1.3-1.9$ \\
\hline & & 1.6 & $1.7-2.3$ \\
3 & 505 & 2.0 & $2.4-3.3$ \\
\hline
\end{tabular}

I. Time points I, 2 and 3 =baseline, one-year follow-up and five-year follow-up.

TOTNAME, total number of named social contacts in previous month; SUMFREN, number of contacts identified as friends; SUMCONF, number of confidants.

Table. 4 Estimated values over time for variables from the Environmental Index (EI) and the Patient Attitude Questionnaire

\begin{tabular}{|c|c|c|c|}
\hline Time point ${ }^{1}$ & $n$ & & $95 \% \mathrm{Cl}$ \\
\hline & & El total score & \\
\hline I & 661 & 25.7 & $25.2-26.2$ \\
\hline 2 & 564 & 8.4 & 7.7-9.1 \\
\hline \multirow[t]{2}{*}{3} & 442 & 10.0 & $9.1-11.0$ \\
\hline & & STATQUO \% positive ${ }^{2}$ & \\
\hline I & 514 & 29.8 & $26.0-33.9$ \\
\hline 2 & 362 & 83.6 & $79.4-87.0$ \\
\hline \multirow[t]{2}{*}{3} & 274 & 83.8 & 78.9-87.7 \\
\hline & & HELPMED \% helpful ${ }^{3}$ & \\
\hline I & 527 & 52.5 & $48.3-56.6$ \\
\hline 2 & 399 & 67.5 & $62.8-71.8$ \\
\hline 3 & 300 & 66.3 & $60.8-71.4$ \\
\hline
\end{tabular}

I. Time points I, 2 and 3 =baseline, one-year follow-up and five-year follow-up.

2. Patient Attitude Questionnaire: \% who wished to stay in current residence.

3. Patient Attitude Questionnaire: \% finding medication helpful.

\section{Environmental Index}

The score on the Environmental Index was analysed (see Table 4). The average number of rules and regulations in the hospital wards was 26 , compared with 10 in the community houses after five years, a highly significant difference.

\section{Patient Attitude Questionnaire}

Two variables from this schedule were analysed: the patient's wish to stay in their

current residence (STATQUO), and their view of how helpful they found their medication (HELPMED) (see Table 4). Whereas $30 \%$ of patients questioned while in hospital wished to remain there, when they were in their community homes the proportion who wanted to stay had risen dramatically to $84 \%$ at both time points. There was a large and significant rise in the proportion of patients who viewed their medication as helpful, which was apparent at one year and persisted over the five years.

\section{Physical Health Index}

Two variables from this schedule were analysed: the proportion of patients with incontinence and the proportion with physical immobility. Both problems increased significantly after one year and then even more so by five years (see Table 5). These increases were strongly associated with patients' age. Patients who developed incontinence were significantly older than those who remained continent (59.6 v. 49.4, $t=5.44$, d.f. $375, P<0.001)$. Those whose mobility became impaired were also significantly older than those who remained mobile (61.1 v. 49.6, $t=5.73$, d.f. 328, $P<0.001)$.

\section{Relationship between baseline variables and five-year outcome}

For every outcome variable investigated, the greatest contribution to the variance stemmed from the baseline value of the variable. A relationship between change over five years and other variables was only evident if the baseline score for that change was controlled for. The results may be summarised as follows (details of the statistical findings as available from the first author upon request).

Age was the most influential baseline variable, in that older patients showed more improvement in PSE total score, but smaller increases in the number of friends and confidants and in BELS domestic and community skills. A higher baseline PSE score was related to greater increases in BELS scores for domestic and community skills, whereas a higher SBS score was related to a smaller increase in BELS domestic skills. Patients with more friends at baseline experienced a greater reduction in EI score and in SBS score, while living in staffed homes was related to a lesser reduction in EI score and SBS score. Both living in private accommodation and in larger households were associated with a lesser reduction in EI score.

\section{DISCUSSION}

\section{Interpretation of the findings}

The modelling exercise using the general estimating equations approach proved to be very successful in revealing the pattern of changes in the data over time. However, there are a number of sources of variation in the data which need to be considered when interpreting these results. 
Table 5 Estimated values over time for variables from the Physical Health Index

\begin{tabular}{lccc}
\hline Time point $^{\prime}$ & $n$ & & $95 \% \mathrm{Cl}$ \\
\hline & & Incontinence, \% & \\
1 & 657 & 10.7 & $8.5-13.3$ \\
2 & 514 & 19.6 & $16.4-23.2$ \\
3 & 425 & 26.8 & $22.9-31.1$ \\
& & Impaired mobility, \% & \\
\cline { 2 - 4 } 2 & 657 & 6.7 & $5.0-8.8$ \\
3 & 512 & 12.6 & $10.1-15.8$ \\
\hline
\end{tabular}

I. Time points I, 2 and 3=baseline, one-year follow-up and five-year follow-up.

A very high percentage of data is missing, particularly at the five-year follow-up, mainly due to the 126 patients who died within five years of discharge. The GEE analysis is based on the assumption that subjects with missing data are representative of the whole sample. Since older patients are more likely to die than younger patients, this assumption is not fully met. The second most common source of lost data is patients who refused to complete certain schedules. The SNS was particularly subject to this problem, data being obtained for only $286(53 \%)$ of the 523 patients followed up at five years. Data obtained by interviewing the staff with the SBS showed that patients who refused to complete the SNS were less sociable than those who complied. Hence, the SNS findings give a falsely optimistic view of the improvements in patients' social relationships when generalised to the whole sample.

Another point to take into account is that the mean estimates do not reflect the considerable variation in individual observations that was noted for most variables. For example, although the mean total score on the PSE did not change over time, individual patients showed marked fluctuations between the three time points. There was also a clear tendency for regression to the mean. Low values at baseline tended to increase over time, while high values tended to decrease. Another source of variation is the number of interviewers and observers involved collecting the data. Over the 13 years of the study, dozens of researchers were trained in the interview techniques and interviewed both staff and patients. At one time TAPS was employing four new assistant psychologists each year. Considerable pains were taken to ensure that the training course produced a high level of reliability, and this was sustained throughout the study by fortnightly joint interviewing and rating sessions. The schedule requiring the most sophisticated judgements is the PSE, and it is noteworthy that no overall changes over time were revealed by the analysis in either the total score or the three sub-scores. This indicates that the interviewers were not introducing a systematic bias.

Informants in the hospital differed from those in the community homes, and it was not feasible to conduct inter-informant reliability tests in the course of the study. Therefore, differences in the perceptions of the informants might have contributed to the differences observed over time. The two most important schedules that rely on information from staff members are the SBS and the BELS, which covers similar types of behaviour to the SBS. It is revealing that the SBS showed no change over time whereas the two scores of the BELS that were analysed showed different patterns of change from baseline through the two follow-ups. Once again this suggests that no systematic bias was operating.

\section{Clinical outcome}

Both the mean total score on the PSE and the mean sub-scores were remarkably stable over the five years of the study. Given that one-third of the study sample were new long-stay patients who were at a relatively early stage of their illness, considerable fluctuations in symptoms would have been expected. As emphasised above, such fluctuations were apparent in the records of individual patients, but were swamped by the stability of symptoms of the majority. We can conclude that the group as a whole showed neither a deterioration nor an improvement in symptoms over the five-year follow-up.

\section{Social outcome}

There were no significant changes over time in the SBS total score or the sub-score on negative symptoms. This result could be seen as disappointing, but it needs to be emphasised that the SBS does not cover many of the types of behaviour that become relevant only when patients are living in the community. Furthermore, the types of behaviour reported by staff and recorded by the SBS are largely equivalent to PSE symptoms. Hence the lack of change in the SBS scores echoes the stability of the PSE.

In contrast to the SBS, significant changes occurred in sub-scores of the BELS. In general the BELS enquires about activities for which there is little or no opportunity in hospital. The scores for community skills increased significantly during the first year following discharge and were sustained up to the five-year follow-up. This indicates that patients had greater access to public facilities such as the post office, cinemas and pubs, and learned to use them. This section of the BELS also covers the use of public transport and the ability to collect social security benefits. Domestic skills include cleaning personal space and communal areas, and the purchase and preparation of food. These also increased significantly during the first year, but fell back over the next four years, although not to the baseline level. There are two possible reasons for this slippage. (a) During the follow-up period, due to advanced age and physical incapacity, some patients were transferred to nursing homes in which there were no opportunities for domestic activities. (b) A second possible reason, given anecdotally, is that staff lost patience with the patients' slowness and inefficiency and took over many of the domestic chores themselves.

Although the patients' social networks did not increase in size, the gain in friends noted at one year was sustained over five years. In addition there was a gain in the number of confidants between one and five years. It makes sense that it took longer to develop intimate relationships than to make new friends. These beneficial changes must be tempered by the fact that at five years we have SNS data on fewer than half of the original sample, and that patients who did not provide data were more socially disabled than those who did. Furthermore, relatively few patients developed social relationships 
with members of the public. We have shown that a local education campaign can increase social contacts between patients and their neighbours (Wolff et al, 1996) and we would strongly recommend this as a new way of combating isolation within the community.

The amount of freedom patients enjoyed in the community homes increased dramatically compared with the hospital wards, with a reduction in the average number of restrictions from 26 to eight in the first year. There was a small increase to an average of 10 by the fifth year, but this still represents a completely different atmosphere from the wards, and refutes the suggestion that mini-institutions were re-created in the community. The patients greatly preferred life in the community homes, with $84 \%$ wishing to remain where they were at both follow-ups. Most of the others wanted to move to a flat of their own, while only a handful said they preferred to return to the hospital.

There was a highly significant increase in the proportion of patients who experienced their medication as helpful, which was sustained over the whole follow-up period. The reason for this change is not clear, since medication regimes remained substantially the same. It could possibly be due to the fact that many patients in the community received their medication from their general practice instead of the psychiatric services, the former being less associated with compulsion.

\section{Physical health}

There was a steady increase in incontinence and physical immobility over the five years of follow-up, both of which were associated with the older patients. Although physical immobility is clearly a consequence of ageing, this is not necessarily true of incontinence, and we noted that some patients who were incontinent in hospital became continent in the community. This suggests that some of the increase in this problem was functional and hence remediable by behavioural approaches.

\section{Interpretation of changes over time}

We investigated how far measures made on the patients in hospital were associated with their outcome five years after discharge. The strongest association by far of any measure was with its baseline value. Other than that, patients' age was linked

\section{CLINICAL IMPLICATIONS}

The quality of life of long-stay patients is considerably better in the community than in the psychiatric hospital, although relatively few became socially integrated.

Patients much prefer living in their community homes to living in the hospital.

Despite a high proportion of patients having active delusions and hallucinations, they are able to acquire skills useful for life in the community.

\section{LIMITATIONS}

There was considerable loss of data over the five-year follow-up due to deaths and refusals by patients.

Differing perceptions of informants in hospital and in the community might have influenced the results.

- The results are only generalisable to well-planned and well-resourced reprovision programmes.

JULIAN LEFF, FRCPsych, Institute of Psychiatry, London; NOAM TRIEMAN, MD, Department of Psychiatry and Behavioural Sciences, Royal Free and University College Medical School, London

Correspondence: Professor Julian Leff, Institute of Psychiatry, De Crespigny Park, Denmark Hill, London SE5 8AF

(First received 15 April 1999, final revision 24 August 1999, accepted 26 August 1999)

with outcome. It is not surprising that older patients found it more difficult to make new friends and to learn new skills. It is more interesting to find that their mental state improved more than that of younger patients. This presumably reflects the early turbulent years of a psychotic illness, and is consonant with the findings of long-term follow-ups (Ciompi, 1980; Harding et al, 1987).

It is of interest that the type of accommodation to which patients were discharged was linked with outcome. Patients living in staffed homes experienced less reduction in restrictions and in social behaviour problems. The explanation almost certainly lies in the selection of the more disturbed patients for the most highly supervised homes. The association of private accommodation with greater restrictiveness, however, is unlikely to be due to selection of more difficult patients, and is probably more reflective of staff attitudes. The link between larger homes and more restrictions is probably attributable to the fact that these homes housed the elderly patients.
In the absence of a control group, is it valid to ascribe the changes found over five years to living in the community rather than to the passage of time? The most dramatic changes were the greatly increased freedom in the patients' accommodation and their satisfaction with their homes. These are clearly due to altered living conditions and not to changes over time in the patients' condition. The same is true of their acquisition of domestic and community skills. Older patients benefited less from the other major change, an enrichment of their social networks, so that this is unlikely to be a consequence of time passing. Hence we can be reasonably confident that the improvements in patients' quality of life are attributable to their move from hospital to the community.

\section{ACKNOWLEDGEMENTS}

We are grateful to Chris Gooch for the data analysis reported here. Rowena Kendal and Helen Smith were very efficient administrators of the project. 


\section{REFERENCES}

Bleuler, M. (1972) Die schizophrenen Geistesstörungen im Lichte langjähriger Kranken-und Familien-Geschichten. Stuttgart:Thieme.

Braun, P., Kochansky, G., Shapiro, R., et al (198I) Overview: deinstitutionalization of psychiatric patients: a critical review of outcome studies. American Journal of Psychiatry, 138, 736-749.

Ciompi, L. (1980) Catamnestic long-term study on the course of life and aging of schizophrenics. Schizophrenia Bulletin, 6, 606-618.

Diggle, P. J., Liang, K. \& Zeger, S. L. (1994) Analysis of Longitudinal Data. Oxford: Oxford University Press.

Gebski, V., Leung, O., McNeil, D., et al (1992) SPIDA User's Manual, Version 6. Eastwood, NSW: Statistical Computing Laboratory.

Harding, C. M., Brooks, G.W., Ashikaga, T., et al (1987) The Vermont longitudinal study of persons with severe mental illness, II: Long-term outcome of subjects who retrospectively met DSM-III criteria for schizophrenia. American Journal of Psychiatry, 144, 727-735.
Jones, D. (1993) The TAPS Project. II: The selection of patients for reprovision. British Journal of Psychiatry, 162 (suppl. 19), 36-39.

Leff, J., O'Driscoll, C., Dayson, D., et al (1990) The TAPS Project, 5: The structure of social-network dat obtained from long-stay patients. British Journal of Psychiatry, I57, 848-852.

—, Thornicroft, G., Coxhead, N., et al (1994) The TAPS Project. 22: A five-year follow-up of long-stay psychiatric patients discharged to the community. British Journal of Psychiatry, 165 (suppl. 25), 13-17.

_ , Trieman, N. \& Gooch, C. (1996) The TAPS Project, 33: A prospective follow-up study of long-stay patients discharged from two psychiatric hospitals. American Journal of Psychiatry, 153, 1318-1324.

O'Driscoll, C. \& Leff, J. (1993) The TAPS Project. 8: Design of the research study on the long-stay patients. British Journal of Psychiatry, 162 (suppl. 19), 18-24.

Pickard, L., Proudfoot, R., Wolfson, P., et al (1992) Evaluating the Closure of Cane Hill Hospital. London: Research and Development for Psychiatry.

Thornicroft, G., Gooch, C., O'Driscoll, C., et al (1993)

The TAPS Project. 9: The reliability of the patient attitude questionnaire. British Journal of Psychiatry, 162 (suppl. 19), 25-29.

Trieman, N. \& Leff, J. (1996) The difficult to place patients in a psychiatric hospital closure programme. The TAPS Project, 24. Psychological Medicine, 25, 765-774.

_ , Leff, J. \& Glover, G. (1999) The TAPS Project, 45: The fate of long stay psychiatric patients resettled in the community. British Medical Journal, 319, 13-16.

Wing, J. K., Cooper, J. E. \& Sartorius, N. (1974) The Description and Classification of Psychiatric Symptoms: An Instruction Manual for the PSE and CATEGO System. London: Cambridge University Press.

Wolff, G., Pathare, S., Craig, T., et al (1996) Public education for community care. A new approach. British Journal of Psychiatry, 168, 44I-447.

Wykes, T. \& Sturt, E. (1986) The measurement of social behaviour in psychiatric patients: an assessment of the reliability and validity of the SBS schedule. British Journal of Psychiatry, 148, I-II.

Zeger, S. L. \& Liang, K. Y. (1986) Longitudinal data analysis for discrete and continuous outcomes. Biometrics, 42, $121-130$ 